San Jose State University SJSU ScholarWorks

Faculty Publications

Industrial and Systems Engineering

January 1995

\title{
Stage Definition for AHS Deployment and an AHS Evolutionary Scenario
}

Jacob Tsao

San Jose State University, jacob.tsao@sjsu.edu

Follow this and additional works at: https://scholarworks.sjsu.edu/indust_syst_eng_pub

Part of the Industrial Engineering Commons, and the Systems Engineering Commons

\section{Recommended Citation}

Jacob Tsao. "Stage Definition for AHS Deployment and an AHS Evolutionary Scenario" Intelligent Vehicle Highway Systems Journal (IVHS Journal) (1995): 359-382.

This Article is brought to you for free and open access by the Industrial and Systems Engineering at SJSU ScholarWorks. It has been accepted for inclusion in Faculty Publications by an authorized administrator of SJSU ScholarWorks. For more information, please contact scholarworks@sjsu.edu. 
IVHS Journal, 1995. Vol. 2(4), pp. 359-382 Reprints available directly from the publisher Photocopying permitted by license only
(C) 1995 OPA (Overseas Publishers Association) Amsterdam BV. Published under license by Gordon and Breach Science Publishers S.A. Printed in Malaysia

\title{
STAGE DEFINITION FOR AHS DEPLOYMENT AND AN AHS EVOLUTIONARY SCENARIO
}

\author{
H.-S. Jacob Tsao \\ PATH Program, Institute of Transportation Studies \\ University of California, Berkeley
}

\begin{abstract}
Pros and cons of various mature Automated Highway Systems (AHS) have been a subject of intense study. However. such discussions are nothing but intellectual exercises unless the issue of how to evolve. in a planned and managed fashion, the current highway systems towards these mature AHS is also addressed. Since full functionality of a mature AHS cannot be realized suddenly, discrete functional steps must be identified and optimized. This paper defines an evolutionary stage towards a mature AHS as any discernible functional increment whose realization may encounter considerable difficulties requiring a significant amount of conscious effort to overcome. A good evolutionary scenario consists of stages each of which provides sufficient additional functionality that justifies the required effort to overcome the associated difficulties. Six dimensions of deployment difficulties are identified: technology, infrastructure, human factors, vehicle manufacturing and maintenance, insurance and public will. An illustrative evolutionary scenario is also provided. Since issues regarding deploying AHS in the real norld actually dictate AHS technological requirements. deployment research should be an integral part of AHS concept definition/evaluation and system design.
\end{abstract}

Key uords: automated highway systems, deployment, evolution, concept definition

\section{INTRODUCTION}

The concept of automated highway systems (AHS) has the potential of offering large capacity and safety gains without requiring a significant amount of right-ofway acquisition. The concept of highway automation began decades ago. (See, for example, [TRB, 1976; Elias et al., 1977; Shladover, 1979].) It has received renewed attention recently due to the fast-worsening problem of urban highway congestion and the belief that integration of advanced sensor, communication, computer and control technologies can safely reduce the average spacing among vehicles at high speed.

In a recent comprehensive treatment of conceptual AHS design, Stevens [Stevens, 1993] discussed AHS deployment and operations goals, analyzed AHS characteris- 
tics and identified 37 alternative AHS concepts. With a narrower scope, Tsao et al. [Tsao et al., 1993(a)] recently identified many major design options and issues for operating fully automated AHS. They also addressed the impacts of the options on major AHS performance criteria including safety, capacity, human factors, infrastructure, cost, etc.

Mostly due to the AHS Precursor Systems Analyses (AHS/PSA) [FHWA, 1992] and the congressional mandate of the Intermodal Surface Transportation Efficiency Act of 1991 (ISTEA), systems research for AHS has enjoyed a recent surge of attention. A large number of AHS operating scenarios have been developed. However, the vast majority of the scenarios did not address how to evolve the current highway systems toward mature AHS. Pros and cons of various mature AHS have been a subject of intense study. However, such discussions are nothing but intellectual exercises unless the issue of how to evolve the current highway systems towards these mature AHS is also addressed. There exist a large number of different possible mature AHS. The additional dimension of evolution leads to an even larger number of possible evolutionary scenarios. This paper proposes an approach to defining evolutionary scenarios and illustrates it with an example.

Al-Ayat and Hall [Al-Ayat and Hall, 1994] developed a framework for planning the evolutionary deployment of all IVHS technologies and provided examples of evolutionary deployment sequences. With a focus on AHS, Hall and Tsao [Hall and Tsao, 1994] recently identified many potential AHS feasibility issues. Design of AHS deployment sequences at this stage is a difficult task because of (a) the sheer large number of possible evolutionary AHS operating scenarios, (b) the existence of many technical and non-technical issues and uncertainties and (c) the difficulty in predicting scenario performance and acceptability under these uncertainties.

On the highest level, the process of AHS deployment can be viewed as overcoming various difficulties in exchange for the provision of desirable AHS functions and user services. Since what is desired of AHS is its functionality or utility (personal or societal), not the enabling technologies, this paper stays on the functional level and discusses only the evolution of automation functions. Since full functionality and user services of a mature AHS cannot be realized suddenly, discrete functional steps must be identified and optimized. This paper defines an evolutionary stage towards a mature AHS as any discernible functional increment whose realization may encounter considerable difficulties requiring a significant amount of conscious effort to overcome. A good evolutionary scenario consists of stages each of which provides sufficient additional functionality and user services that justify the effort required for overcoming the associated difficulties. Six dimensions of deployment difficulties as well as specific difficulties are identified. The six dimensions are technology, infrastructure, human factors, vehicle manufacturing and maintenance, insurance and public will.

Initial AHS market penetration could be the most difficult stage of all. However, the presence of many difficulties imply that there exist many constraints on initial AHS deployment and there may not be many choices. With this observation, Tsao [Tsao, 1995(a)] recently identified seven major groups of constraints on initial AHS deployment, where an initial AHS user service is defined to be any service that involves hands-off and feet-off driving. He also proposed a freeway shuttle van/mini- 
bus service for AHS debut. Comparison of the desirability of the different mature AHS is also difficult, at least at this time. Given a feasible initial AHS deployment strategy and a target mature AHS, an evolutionary scenario can be viewed as a collection of intermediate stages, possibly overlapping and parallel, connecting the two ends.

For illustration, the initial deployment strategy of automated freeway shuttle service proposed by Tsao [Tsao, 1995(a)] recently is adopted and a particular mature urban AHS is selected as an example. An illustrative evolutionary scenario is then defined as a sequence of stages connecting the two ends. The functional increments and the difficulties associated with each step are also discussed.

As part of the Precursor Systems Analyses of Automated Highway Systems (AHS/ PSA) effort [FHWA, 1992], Ward [Ward, 1994] proposed an evolutionary scenario. He discussed in detail and focused primarily on the evolution from today's highway systems towards an AHS where the automated automobiles are mixed with the manual vehicles in the same lane, are capable of driving themselves along a lane, and have the capability to form close-spaced platoons spontaneously with longitudinally adjacent automated automobiles, if any. The different stages were motivated primarily by the incremental advances of AHS technologies. While some issues associated with the proposed staged evolution were discussed, he focused primarily on cost-benefit and acceptance, particularly from the automobile purchaser point of view. The possibility of automating other vehicle types, e.g. transit vehicles, was not considered. "Fail-safety" and "fail-softness" were assumed, which simplified his discussion of AHS deployment. This paper seeks to identify possible stages beyond automated driving along a lane. It does consider the possibility of accommodating multiple vehicle types on AHS but does not assume fail-safety and fail-softness for the early deployment stages. It considers not only the technological (deployment) issues but also issues in five other dimensions.

This paper is organized as follows. Section 2 briefly describes six dimensions of deployment difficulties. Section 3 first defines what qualifies as an AHS deployment stage towards a mature AHS and then introduces an approach to identifying AHS evolutionary scenarios. Section 4 describes the illustrative evolutionary scenario for an urban AHS. Concluding remarks are given in Section 5.

\section{SIX DIMENSIONS OF DEPLOYMENT DIFFICULTIES}

The difficulties of AHS deployment are grouped in the following six dimensions: (D1) technology, (D2) infrastructure, (D3) human factors (user-vehicle-system interface), (D4) vehicle manufacturing and maintenance, (D5) insurance, and (D6) public will.

\section{Technology}

Major sources of deployment difficulties in the technology dimension include (D1.1) vehicle diversity, (D1.2) automated (driving) functions, (D1.3) technology maturation, and (DI.4) functional diversity. 
(D1.1) Vehicle Diversity (Accommodation Scope). Vehicle diversity (or accommodation scope) refers to the types of vehicle to be automated on AHS. We, in this paper, consider automation for multiple vehicle types, not just automobiles. Vehicle uniformity makes control of vehicles and AHS simpler and likely safer.

(D1.2) Automated (Driving) Functions. Automated functions are the driving tasks that are automated and refer to the degree of driving automation (or the automation capabilities). Like many other technologies, automation technologies as well as the associated manufacturing and maintenance technologies will advance gradually. Faced with the uncertainty of market penetration, industrial investment in research, development, marketing and manufacturing may be gradual. Therefore, initial deployment is likely to consist of simple and yet useful user service. Based on earlier successes as well as public acceptance, technologies will then be further developed, refined and proven. In other words, automation functions will be incrementally deployed. This characteristic could impact the whole AHS evolution process. In this subsection, we concentrate on the functions provided by the sensing and communication technologies. Those provided by the computers and actuators, although vital for automation, need discussion in a more technical setting.

Major functional steps provided by the communication technologies include: (a) no communication capability on the vehicle, (b) communication (i.e. information exchange) between vehicles only, (c) communication between vehicle and roadside only, and (d) communication between vehicles as well as between vehicle and roadside. Sensing functions, when combined with communication technologies, can be expected to provide the following functional increments, among others, for highway automation: (i) providing sufficient information about the traffic and obstacles ahead in the same lane for automated driving along a lane so that the probability of collision with a vehicle or an obstacle ahead, fully or partially in the same lane, is minimized; (ii) providing sufficient information about the traffic on the neighboring lanes as well for safer automated driving along a lane so that early warning and reaction can be made about accidents spilling over from neighboring lanes or about the potential of abrupt invasion by vehicles from neighboring lanes, (iii) providing sufficient information about the traffic on the neighboring lanes for safe automated lane changing; (iv) providing sufficient information for automated merging and diverging of traffic at specified locations. Note that the provision of these functions in mixed traffic is much more difficult than its counterpart in automated traffic that is physically segregated from the manual traffic.

(D1.3) Technology Maturation. Technology maturation refers to the progressive process of an automation capability to physically function as conceptually intended. It also refers to fail-safety and fail-softness. (Note that both of them can also be viewed as automation functions.) Vehicle and system failures do occur and fail-safety and "failsoftness" are assumed to be reached only gradually.

To ensure safe automated driving, early-generations of automation-equipped vehicles may need to be inspected and maintained frequently and rigorously. Before automated vehicles are made fail-safe, driver training for handling emergency may be required. 
(D1.4) Functional Diversity. Automation functions will likely be deployed incrementally. Therefore, at any point in time, there are likely multiple classes of automation-equipped vehicles each of which is capable of a particular set of automation functions. In other words, automation functionality will likely vary from vehicle to vehicle. Therefore, a stringent requirement for any stage of the AHS deployment may be to support vehicles with varying automation capabilities. For example, it may be required to support both autonomous vehicles (without communication capability) and those vehicles with the close-spaced platooning capability (with, among other things, additional capability of communication). Note that in this paper close-spaced platooning refers to the operating concept where vehicles travel in clustered formation. In other words, intervehicle spacing is either very short or very long and the short spacing is assigned to maximize capacity and to minimize the relative speed at collision if a collision does occur after a failure.

The existence of a large variety of vehicle automation capabilities may cause difficulty in vehicle operation. For example, a platooning-only AHS is infeasible if a large percentage of automation-equipped vehicles are "autonomous vehicles" and do not have any communication capability. Therefore, a small number of distinguishable levels of automation capability may be highly desirable for AHS operation. Note that different automation technologies could support a common driving function. Furthermore, completely different technology approaches may provide complete automation of all driving tasks. There may even be the issue of technology diversity. For example, different geographical areas may implement AHS concepts differently and different vehicle manufacturers may use different vehicle automation technologies. However, since this paper concentrates on the function level, it does not deal with the possible issue of technology diversity. Another reason behind this non-treatment is that a national architecture is expected to set the technology standards for nationwide AHS compatibility.

\section{Infrastructure}

There are at least five sources of difficulties in the infrastructure dimension: (D2.1) support of automated functions, (D2.2) modification and construction, (D2.3) usefulness of modification for the step itself, (D2.4) cost and financing, and (D2.5) rate of modification.

For developing alternate IVHS deployment strategies, Al-Ayat and Hall [Al-Ayat and Hall, 1994] adopted six guidelines, including (i) functionality provided at each step is useful by itself and the usefulness does not require full deployment of subsequent steps and (ii) each deployment step has a high likelihood of acceptance by the user. Item (i) implies that even if deployment is halted, the deployed functionality should continue to provide useful service. These two guidelines are particularly important for infrastructure modification. Category (D2.3) refers to these two guidelines.

The functional steps supported by the infrastructure include: (i) a continuous lane on one highway with sufficient support for automated driving, (ii) such a lane on one highway and onto another, i.e. one that allows continuous automated driving from one highway to a crossing highway, (iii) a network of such lanes with sufficient 
support for continuous automated driving across different highways, (iv) a network of such lanes with special on-ramps and off-ramps dedicated to the use by automation-equipped vehicles and (v) a network of such lanes that are physically segregated from the manual traffic.

\section{Human Factors (User-Vehicle-System Interface)}

This dimension includes the following difficulties: (D3.1) transitional tasks, (D3.2) driver monitoring during automated driving, (D3.3) emergency maneuvering, and (D3.4) discomfort. Note that users include the drivers as well as the passengers. Passengers may include those traveling on automobiles as well as those on transit vehicles.

Tsao et al. [Tsao et al., 1993(b)] identified many possible human factors issues for normal AHS driving. Resuming manual control of the vehicle after a long period of fully automated driving is a new task for drivers. It is possible that initial automation technologies, due to cost and other constraints, cannot offer user-friendly transitions. Consequently, additional driver skills may be required.

Human errors account for about $90 \%$ of the current highway trafic accidents and vehicle/highway automation has the potential of eliminating all accidents caused by driver errors. However, such automation requires additional equipment on the vehicle as well as on the roadside and could introduce new kinds of safety hazards. Before the maturation of these automation technologies, the driver may be required to play an active supervisory role monitoring the operation of the automated vehicle. Tsao et al. [Tsao et al., 1995] identified many possible AHS failure events that might require human intervention in vehicle/system operation for safety, especially during the early stages of deployment when the automation technologies have not been perfected yet. Emergency handling could also be part of the new driver role.

The requirement for transitional skills, the monitoring role and the emergencyhandling responsibility may necessitate driver training, which is not likely to entice car owners to purchase automation options. In fact, it is possible that, during initial deployment, only trained professiona/s, e.g. professional drivers with additional AHS training and credential, would be qualified to invoke automated driving.

\section{Vehicle Manufacturing and Maintenance}

Major difficulties that have to be overcome include: (D4.1) manufacturing commitment, i.e. commitment of automakers to manufacture and service automationequipped vehicles, and (D4.2) vehicle costs, particularly the purchase and maintenance costs of automation-equipped vehicles.

(D4.1) Manufacturing Commitment. The automakers will not commit their resources to making and servicing automation-equipped vehicles unless potential liability issues can be resolved and there is a profit to be made. It is well-known that, at the present time, a full-scale deployment of AHS technologies is full of uncertainties. To enter the business of making automation-equipped vehicles, they may prefer to start 
with a smaller but less uncertain niche than a much bigger but very uncertain market. Therefore, identification of an initial niche vehicle market for the automakers could be crucial. Same can be said about their search for subsequent markets.

(D4.2) Vehicle Costs. Before wide public acceptance, the vehicle costs, including manufacturing and maintenance, could be very high. This may hinder market penetration and may also violate a major concern of equity of use.

\section{Insurance}

Given the possible high degree of interdependency among vehicles, roadway support, and roadside intelligence for safe AHS operation, a clear definition and distribution of liability is required but could be challenging. To insure against liability, the difficulties include: (D5.1) the commitment of insurance industry to carry liability, including tort, product, and government liability and (D5.2) cost of insurance.

(DS.I) Commitment by the Insurance Industry. Even today, in many States, it is a legal requirement that each vehicle be insured for liability. This requirement will most likely remain after AHS deployment, if not made more stringent. Therefore, AHS will not survive if the insurance companies refuse to issue policy. AHS R\&D community must take into consideration the interest and the attitude of the insurance industry in designing deployment strategies. Incremental introduction of automation features that have proven safe may be required. Frequent and rigorous vehicle inspection and maintenance may also be required, at least initially.

(DS.2) Insurance Costs. Suppose that liability insurance will become available initially. Upon introduction of automated driving on highways, premium and/or deductible may be too high for individual owners of automation-equipped vehicles. However, fleet operators could afford it more easily and distribute the additional cost to the individual service users. Without liability insurance, owners of automation-equipped vehicles have to be self-insured. But, it is likely that such self-insurance is allowed only for large businesses or government agencies.

\section{Public Will}

This dimension contains at least following sources of difficulties: (D6.1) user service and cost, (D6.2) user safety and perceived safety, (D6.3) societal service, (D6.4) societal cost, (D6.5) environment impact.

AHS research and development community must strive to win the acceptance by various interest groups and eventually the general public. It could win their support by offering products that appeal to them, particularly in terms of user service [Bishop et al., 1994], safety, perceived safety, comfort, convenience, reduced travel delays, cost and environment impact. However, that may not be sufficient; we need to learn from the failures and successes experienced by other industries in introducing new prod- 
ucts. Tsals et al. [Tsals et al., 1993] studied four examples (Global Positioning System, Bar Coding, Nuclear Power and Magnetic Resonance Imaging) and argued for the necessity to be (a) forthright with eventual customers about benefits and drawbacks of new technologies and (b) sensitive to public perception of new technologies (which may be different from reality).

To ensure achieving the full potential of automation, we should assume that interest groups and general public may reject AHS quickly but would accept it only gradually and incrementally. (Interest groups represent the public, only to some degree.) Stages of deployment must be carefully determined and implemented so that interest, trust and continued support by the general public can be cultivated.

\section{A STAGE DEFINITION APPROACH}

Qualifications of an AHS deployment stage, i.e. the criteria for judging whether an incremental step in AHS deployment deserves to be designated as a deployment stage, have to be clearly defined and justified. We propose the following guideline. An incremental step in AHS deployment can be considered as an AHS deployment stage if and only if it is a discernible incremental functional step whose realization may encounter considerable difficulties requiring a significant amount of conscious effort to overcome. (This definition is based on difficulty of realizing a function, rather than the function itself, because if its realization involves no difficulty at all, making the functional increment a separate deployment stage would only complicate the process of designing deployment strategies.) The utility of automation functions is judged according to public will, i.e. the desires of the driving population and the general public. The possible difficulties include those in the following six dimensions: technology, infrastructure, human factors, vehicle manufacturing and maintenance, insurance and public will. Smallest functional increments possibly incurring any type of difficulty that requires conscious effort to overcome should be sought. Some stages may be skipped if the difficulties turn out to be minor and can be easily overcome. In this way, it is hoped that no major stages will be neglected.

Due to the existence of many uncertainties, sequencing stages for successful deployment is difficult. Timing of deployment stages is even more difficult and is out of the scope of this paper. However, under the guidance of a deployment sequence, the task of timing may become easier.

Since many possible enabling technologies exist, for ease of discussion, we address deployment of AHS functions without specifying the enabling technologies. The impact of the enabling technologies on deployment scenarios is by itself a challenging subject of research. The functional approach is also justified by the fact that highway automation is needed to serve society's transportation needs and those needs are usually translated into vehicle and highway functions, without even referring to the enabling technologies.

Given the existence of many possible mature AHS [Tsao et al, 1993(a); Tsao et al., 1993(b); Stevens, 1993; Stevens, 1994] and the many difficulties discussed above, design of evolutionary scenarios is particularly difficult. Tsao [Tsao, 1995(a)] observed that particularly important and difficult in defining a deployment sequence is 
the very first step, i.e. the first user service involving fully automated freeway driving (hands-off and feet-om). He also observed that this importance and the difficulty imply that many factors may severely constrain the initial deployment and there may only be few choices. To facilitate the design of deployment stages towards a mature AHS, we adopt the approach of identifying good initial AHS deployment targets and then "building up" the intermediate stages between an initial target and the mature AHS system. Note that a good initial AHS target should not constrain the AHS development and deployment in such a way that some major mature AHS cannot be evolved from the initial deployment. It is also this approach that motivated the work of Tsao [Tsao, 1995(a)].

\section{AN EVOLUTIONARY SCENARIO FOR AN URBAN AHS}

It is helpful to point out at the outset the initial deployment strategy and the mature urban AHS that the evolutionary steps are designed for. The initial deployment strategy of a freeway shuttle van service will be summarized later in this section. We now describe some key features of the mature urban AHS as follows.

\section{A Mature Urban AHS}

The key features are posed as assumptions on the future AHS. They are grouped in six different difficulty categories.

(MAI) Technology. Multiple vehicle types are supported on the mature AHS, including automobiles, transit vehicles and trucks. For simplicity, we concentrate on automobiles and transit vehicles in the rest of this section. We assume a vehicle-centered platooning technology, where the qualifier vehicle-centered is used in a loose way to signify heavy reliance on the vehicle intelligence, rather than the roadside intelligence, for safe operation. Support from the infrastructure may be required but the actual requirement depends on the actual automation technology and is out of the scope of this paper.

Four different grades of automation-equipped vehicle are summarized in Table 1, where any vehicle of any particular grade possesses all the automation capabilities associated with all the lower grades. For convenience, non-platooning-equipped vehicles will be called loners. A vehicle traveling alone without being part of any closespaced platoon is said to travel in solitude. A vehicle traveling in solitude can either be a platooning-equipped vehicle or a loner. Note that a loner vehicle refers to an automation-equipped vehicle that cannot cooperate with other vehicles to form a platoon. In other words, loner is an attribute of a vehicle while solitude is an attribute of how a vehicle travels at particular points in time. All non-automobile automation-equipped vehicles are loner vehicles. Automation-equipped automobiles may or may not be platooning-equipped.

(MA2) Infrastructure. Automated traffic is physically and completely separated from 
Table I. Increasing Grades of Automation-Equipped Vehicles

\begin{tabular}{ll}
\hline Grade & Description \\
\hline$G 1$ & Automated Lane Cruising (Transit Vehicles Only) \\
& - Lane Keeping \\
& - Vehicles Following \\
& - Speed Limit Observation \\
& (Dependent Upon Roadway Geometry, etc.) \\
& - Safe Speed Determination \\
& (Dependent Upon Weather/Driving/Traffic Conditions, etc.) \\
& - Obstacle Detection and Avoidance \\
& $\begin{array}{l}\text { Fail-Safe Automated Lane Cruising } \\
\text { (Vehicle Brought to a Safe Stop After Safety-Critical Failure) }\end{array}$ \\
G2 & $\begin{array}{l}\text { Fail-Safe Automated Lane Changing/Merging/Diverging. } \\
\text { (Communication Capability Assumed) }\end{array}$ \\
& Fail-Safe Automated Platooning \\
G4 (Automobiles Only)
\end{tabular}

the manual traffic. The AHS consists of a dedicated network of automated urban highways that is at grade level, occupying inner lanes of highway, and basically within the current right-of-way. There are no barriers between any two automated lanes.

Special on-ramps and off-ramps (in addition to the current manual on-ramps and off-ramps) provide direct access to and egress from the automated lanes via highway median. (These may be very costly, in terms of both construction costs and possible right-of-way acquisitions.) Special highway-to-highway connector ramps (in addition to the current manual connector ramps) provide direct connection between automated lanes. Vehicle check-in is performed at high speed without stopping and minimum amount of additional real estate is required to accommodate check-in facilities at the automated on-ramps.

An automated highway may have multiple automated lanes and the number of automated lanes vary with highway section. On those automated highways with only one automated lane (the left-most lane), all types of automated vehicles share that lane and platooning-equipped vehicles travel in "spontaneous platoons." On twolane automated highways, the second automated lane (i.e. the second lane from the median) is dedicated to the automobiles while the first automated lane (i.e. the leftmost lane) is shared by all vehicle types. Vehicles on the first automated lane travel without close-spaced formation. In and only in sections of automated highways with high demand of usage and only during time of high demand, the second automated lane is dedicated to the use by platooning-equipped automobiles and they travel in close-spaced platoons. The illustrative evolutionary scenario will feature a mature urban AHS with two automated lanes.

On automated highways with more than two lanes, the innermost automated lane is dedicated to the dominant vehicle type-automobiles-while the outermost lane is shared by all vehicle types. The use of the lanes in between could vary according to the types of vehicles supported and the amount of traffic of those types. 
Complete segregation of different types of automated traffic is very difficult, if not impossible, because construction of separate access/egress ramps and that of separate connector ramps at highway-to-highway interchanges will likely require additional real estate and additional large structures.

(MA3) Human Factors. The driver of an automation-equipped automobile has the right to determine if he/she wants to platoon or not. (But, the driver may have to pay more for solitary travel because of less effective use of infrastructure capacity.) If so, the vehicle, depending the trip length and traffic condition, may be automatically driven into the second automated lane and join the platooning traffic on the second automated lane. If the driver does not feel comfortable with close-spaced platooning, he or she will travel on the first automated lane throughout the trip. The overall interface between driver (as well as passengers) and vehicle/system is assumed userfriendly.

(MA4) Vehicle Manufacturing and Maintenance. Vehicle manufacturers can and do manufacture aftordable, reliable and fail-safe automation-equipped vehicles. Such vehicles can also be maintained properly, conveniently and affordably.

(MA5) Insurance. Liability insurance is available at an affordable rate.

(MA6) Public Will. Such a system is accepted and supported by the general public.

Before defining the evolutionary stages, we point out an observation of strategic importance for evolution towards this AHS-the inevitability of transit-vehicle automation. We first make four assumptions: One, at the early stages, there will not be sufficient demand or public will to justify the dedication of one lane for the exclusive use by automated vehicles. Therefore, automated vehicles use only the HOV lane (left-most lane). The safety of such mixing of traffic is assumed. Two, a set of highway-to-highway connector ramps connecting directly the HOV (left-most) lanes of two crossing highways is constructed for each highway-to-highway interchange. Three, the construction of another independent set of highway-to-highway connector ramps for supporting the dedicated use of automated traffic from one highway to the crossing highway is infeasible. Four, when the demand becomes sufficient, the left-most lane (the HOV lane previously) is dedicated to automated traffic. (The second leftmost lane is then dedicated to HOV traffic.) Under these four assumptions, when the demand for automation becomes sufficient to justify the dedication of one automated lane, the HOV highway-to-highway connector ramps must be dedicated to the use by automated traffic and the non-automated HOV users are deprived of the privilege of using any direct highway-to-highway connector ramps. Moreover, if special access/ egress ramps directly connecting the left-most lane to the city streets are also built for HOV and automated traffic at early AHS deployment stages, such deprivation would most likely also apply to these ramps. This may be politically difficult and unlikely to happen, unless a significant percentage of the automated traffic are automated transit vehicles and HOVs. In short, automation of transit vehicles could be crucial for the eventual success of automobile automation because of the fact that, without it, 
Table II. 12 Evolutionary Stages To a Mature Urban AHS

\begin{tabular}{|c|c|}
\hline Stage & Description \\
\hline SI & $\begin{array}{l}\text { The Initial Deployment Strategy: Vans/Mini-buses Providing A Freeway Shuttle Service with } \\
\text { Automated Lane Cruising Supervised by a Professional Driver in Mixed Traflic on HOV Lane }\end{array}$ \\
\hline S2 & $\begin{array}{l}\text { Construction of Highway-to-Highway HOV Connector Ramps and Equipping HOV Lanes } \\
\text { for Automated Driving }\end{array}$ \\
\hline S3 & Achieving Vehicle Fail-Safety \\
\hline S4 & Automation of Automobiles \\
\hline S5 & Dedication of One Automated/Transition Lane for Transition and Automated Driving \\
\hline S6 & Automation of Lane-Changing into and out of the Automated/Transition Lane \\
\hline S7 & $\begin{array}{l}\text { Dedication of One Automated Lane (No Transitioning) and Automation of Merging/ } \\
\text { Diverging at (Automated) Highway-to-Highway Connector Ramps }\end{array}$ \\
\hline S8 & $\begin{array}{l}\text { Construction of Automated On-Ramps and Off-Ramps with Barriers at High-Demand } \\
\text { Locations }\end{array}$ \\
\hline S9 & $\begin{array}{l}\text { Segregation of Automated Trafic from Manual Trafic with Physical Barriers for Safer and } \\
\text { High-Speed Automated Driving }\end{array}$ \\
\hline S10 & Two Automated Lanes for Capacity and Higher Speed on the Second Automated Lane \\
\hline S11 & Automobile Platooning on the Second Automated Lane for Higher Capacity \\
\hline $\mathrm{S} 12$ & $\begin{array}{l}\text { A Mature Urban AHS: A Segregated Automated Highway Network on Existing Right-of- } \\
\text { Way }\end{array}$ \\
\hline
\end{tabular}

the conversion of HOV facilities into automated facilities may encounter fierce resistance.

\section{Twelve Evolutionary Stages}

We now state the evolutionary scenario. It consists of 12 sequential stages with possible overlap between consecutive stages. Each stage provides an increment in AHS functionality and the functional increment is summarized by the title of the stage. Inevitably at this point in time, judgements are made about the functional feasibility and its acceptability. Table 2 summarizes the 12 stages.

To avoid redundant and repeated statements about the commonality among stages, we describe only the functional increments and operational differences from previous stages. However, for the initial and the final stages, we describe the whole functionality and operation. Due to the vehicle-centered nature of the assumed automation technology, the infrastructure is treated as playing a supporting role, although the 
support is an integral part of the automation technology. Nevertheless, for clarity, we state the functions provided by the infrastructure first and then discuss the technology and other dimensions. Automated functions together with the driver roles under the category of human factors specify the degree of automation. Note that we start at the stage where driving along a lane is safely automated in the absence of vehicle failures and sudden intrusion by other vehicles or foreign objects in front of it. We now summarize the stages.

(SI) The Initial Deployment Strategy: Vans/Mini-buses Providing a Freeway Shuttle Service with Automated Lane Cruising Supervised by a Professional Driver in Mixed Traffic on HOV Lane. The automation target at this initial stage is vans and minibuses, instead of automobiles. Figure 1 depicts an example four-lane urban highway and illustrates this initial deployment strategy. (This four-lane highway will also be used to illustrate future stages.) These transit vehicles provide a automated freeway shuttle service between two activity centers that are near freeway entrances and exits, e.g. the airport and the downtown of a metropolitan area. Driving along an HOV lane is automated. (The enabling technology varies. It could include some roadside sensing and intelligence. It could also be residing completely on the vehicle, i.e. autonomous.) Vehicles and roadside system, if any, are not made fail-safe yet. A professional driver with special training is at the driver seat at all times to perform (i) manual driving on city streets, (ii) manual driving from a freeway entrance to the HOV lane next to the median (the left-most lane), (iii) transitional task from manual driving mode into automated driving mode, (iv) supervision during automated driving, including watching out for possible accident spill-overs from neighboring lanes and possible abrupt invasion by vehicles from neighboring lanes, (v) emergency handling, (vi) transitional task from automated driving mode back to manual mode, (vii) manual driving from one highway to a crossing highway (by crossing the slow lanes on both highways).

Automation technologies include automated lane keeping, obstacle detection/avoidance, automated vehicle following, automated speed limit observation and automated determination of safe speed, which may depend on weather, lighting, driving and traffic conditions. Vehicles are frequently inspected at the fleet operator's maintenance facilities (and perhaps under continuous self-monitoring) so that there is no need to have a check-in facility at an entrace. Fleet operators bear the possible high initial cost of purchasing and maintaining automation-equipped vehicles as well as possible high initial cost of insurance. This type of shuttle service expands as infrastructure modification continues. New traffic and liability laws may be required at this very first stage.

(S2) Construction of Highway-to-Highway HOV Connector Ramps and Equipping HOV Lanes for Automated Driving. The major efforts in this stage include the construction of HOV highway-to-highway connector ramps and equipping a network of HOV lanes for automated driving. The goal is to pave the way for a network of HOV lanes sufficiently instrumented for continuous automated driving between any pair of major activity centers in a metropolitan area. A direct benefit is minimization of delay for HOV traffic, which could entice more ridesharing, including the demand for the automated freeway shuttle service. 


\section{Legend $\because 3$ \\ (1) "Waves" Indicate Automation-Equippedivehicles \\ (2) Black Mini-bus is Automaticălly byituen (Hands-off, Feet off) \\ (3) Zigzag Árrows lndicate Communication Difuction \\ (4) Dots at Lane Center Indicate Lan êa Markers \\ (5) Hov Lane is Separated From General-access Lanes by Conspicuous Markings (Not Physical Barrierers) \\ (6) Stràight and Curved Arrows Indicate Direction of Motion}

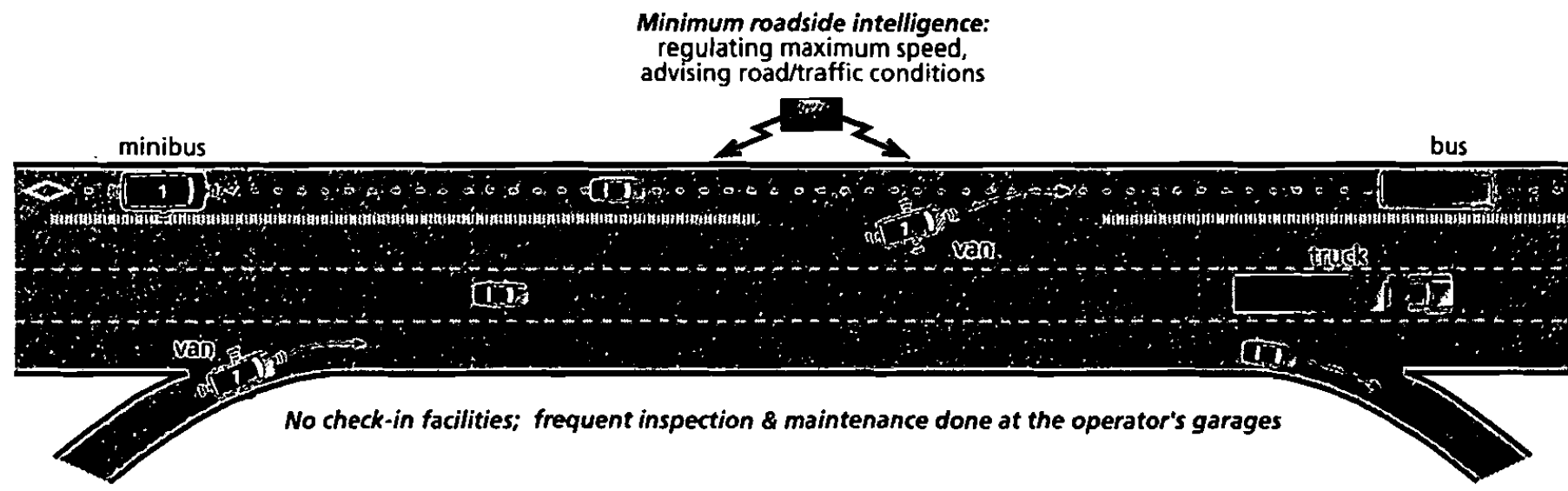

FIGURE 1 (S1) The Initial Deployment Strategy: Vans/Mini-busses Providing a Freeway Shuttle Service with Automated Lane Cruising Supervised by a Professional Driver in Mixed Traffic on HOV Lane. 


$$
\pm
$$


metropolitan area, the overall cost of constructing such connector ramps at all interchanges in a metropolitan area may be moderate. If AHS deployment is halted for some reason, the HOV-to-HOV highway connector ramps remain useful for HOV traffic. Note that, at this stage, lane changing has not been automated and a highway-change requires take-over of manual control by the professional driver before diverging and manual driving through the HOV connector ramp and into the HOV traffic on the crossing highway.

(S3) Achieving Vehicle Fail-Safety. At this stage, the technology for automated lanecruising has matured and become fail-safe. The vehicle will be brought to a safe stop after a safety-critical vehicle failure or if there is safety-impacting debris ahead in the lane. The manual controls of vehicles with the fail-safe feature become non-responsive during automated driving and no driver intervention during automated driving is allowed, except through a procedure of taking over manual control or the use of a "panic button". Due to the fail-safe feature, the professional driver is no longer responsible for monitoring normal vehicle operation. Depending upon the sophistication of the automation technology, particularly the sensing technology, the professional driver may still be responsible for reacting to possible sudden and dangerous movements of the surrounding vehicles. We assume that all future technology increments come with the fail-safe feature.

Note that fail-safety is a general yet stringent requirement that is often interpreted as unconditional safety, even after the occurrence of a failure. Also note that failure is subject to rigorous definition and a failure could be a vehicle failure, a system failure, communication interference, or the presence of debris ahead in the lane. Consequently, the concept of fail-safety is also subject to rigorous definition. Therefore, a broad assumption of fail-safety may be too stringent and perhaps even unrealistic. Detailed definition of failure and fail-safety is beyond the scope of this paper.

The user-friendliness of the transitional tasks is also achieved in this stage. We assume that the user-vehicle-system interface will remain user-friendly for the rest of the deployment stages.

(S4) Automation of Automobiles. Network-wide HOV lane modification for automation and construction of highway-to-highway HOV connector ramps are completed. This provides infrastructure support for automobile automation. With the extensive HOV network, automobile owners can use the automation feature throughout their freeway trips, except a brief resumption of manual control while using the highwayto-highway HOV connector ramps. Due to maturation of the technologies and the fail-safe design, vehicle check-in can be performed at high speed without stopping and minimum amount of additional real estate is required to accommodate check-in facilities at the entrances. Check-in may require only status reporting by the vehicles to the roadside. Because of fail-safety, automobile drivers need not monitor the operation of vehicle during normal automated driving and need not intervene with vehicle operation for emergency handling. Transitional tasks have been made easy through user-friendly user interface. The cost of purchasing, maintaining and insuring automation-equipped automobiles has become affordable. 
(S5) Dedication of one Automated/Transition Lane for Transition and Automated Driving. When the demand for automated driving has reached a certain threshold, the left-most lane can be dedicated to automation-equipped vehicles for transitioning and automated lane-cruising. In other words, the HOV lane is redesignated as the automated/transition lane. The second left-most lane becomes the HOV lane. Since vehicles are not equipped with the automated lane-changing capability, to access the automated/transition lane, they are driven manually into the HOV lane first and then the automated/transition lane and then transition into the automated driving mode. (Diverging and merging at the special highway-to-highway connector ramps is still manual.) HOV traffic travels on only the HOV lane, not the automated lane. Physical barriers separating the automated/transition lane from the HOV lane are erected at highway-to-highway interchanges, particularly at the merge point, to prevent lane changing and possible intrusion by manually-driven vehicles. Instrumenting the new HOV lane for automated driving needs to be completed in this stage.

Note that by dedicating the left-most lane as the automated/transition lane, the previously direct HOV highway-to-highway connector ramps can only be used by automation-equipped vehicles. Since merging and diverging at the ramps are still performed manually, these ramps can be referred to as semi-automated highway-tohighway connector ramps. A potential issue is that, starting at this stage, the HOV traffic is deprived of the use of the now semi-automated highway-to-highway connector ramps.

(S6) Automation of Lane-Changing into and out of the Automated/Transition Lane. The main added function of this stage is vehicle's automated lane-changing capability. Vehicles so equipped can transition between the automated and manual driving modes on the HOV lane and then are driven automatically onto the automated/ transition lane by the newly added automated lane-changing capability. Those not so equipped are first manually driven onto the automated/transition lane and then transition into the automated driving mode.

Assuming that the transition tasks are user-friendly, transition should take very little time and, at any point in time, only a small fraction of vehicles traveling on the automated/transition lane are in the transitioning process. To maximize the safety of lane-changing into the automated/transition lane, it can be stipulated that a vehicle can begin an automated lane-change maneuver into the automated/transition lane only after it has successfully negotiated via communication with the two vehicles adjacent to the intended gap (if they are nearby). Note that the tacit assumption is that negotiation through communication is required for safety and the two vehicles are already under automatic control, i.e. vehicle-to-vehicle negotiation can take place only after the involved vehicles have transitioned into and are under automated control. However, such stipulation is unreasonable for lane-changing out of the automated/transition lane into the HOV lane because not all vehicles on the HOV lane are automation-equipped. Nevertheless, it can be stipulated that before a vehicle can begin the lane-change maneuver from the automated/transition lane into the HOV lane it should notify and obtain consent from the two longitudinally adjacent vehicles in the automated/transition lane (if nearby). In this way, if a vehicle encounters and detects safety hazards while changing lanes from the automated/transition lane 
into the HOV lane, abort can be safer than otherwise due to the full awareness of the two adjacent automated vehicles. Note that in order for a vehicle to change lane into or out of the automated lane, the two longitudinally adjacent vehicles must have already transitioned into the automated driving mode. Otherwise, it has to wait. Because at any point in time only a small fraction of the vehicles are in the transitioning process, the wait should be very brief.

We assume that the automated lane-cruising capability can be upgraded to the automated lane-changing capability. Note that such technological upgradability could be crucial to the success of AHS deployment. As stated earlier, we assume that, at any stage following (S3), fail-safety comes with the added functions.

Merging/diverging is different from regular lane-changing in that the former needs to be completed timely at a specific location while the latter can be aborted and retried at a later time. Therefore, automated merging/diverging could involve more sophisticated technology than automated lane-changing. We assume that the additional sophistication resides on the roadside and not on the vehicle. In other words, any vehicle equipped with automated lane-changing capability can perform automated merging/diverging as long as the roadside (infrastructure) is instrumented to provide the necessary information and intelligence. For convenience, automated lanechanging/merging/diverging capability of a vehicle will be occasionally referred to simply as automated lane-changing capability in the rest of this paper.

(S7) Dedication of One Automated Lane (No Transitioning) and Automation of Merging/Diverging at (Automated) Highway-to-Highway Connector Ramps. In this stage, the roadside (infrastructure) intelligence is upgraded to provide necessary information and guidance for supporting safe automated traffic merging and diverging at the HOV highway-to-highway connector ramps. The old automated/transition lane is redesignated as the automated lane. On the automated lane, there is no manual driving allowed. Only those vehicles equipped with the lane-changing capability can use the automated lane. Automated merging and diverging enable continuous automated driving across different highways. The previously semi-automated highway-tohighway connector ramps are now redesignated as automated highway-to-highway connector ramps. See Figure 3 for illustration.

Note that an automated lane refers to a lane on which all the vehicles are under automated control. This goes beyond the concept of "instrumented lane," which can be interpreted as a lane suitably equipped for automated driving. The HOV lane, for example, can be regarded as an instrumented lane but not an automated lane.

Automation-equipped vehicles transition between the manual and automated modes on the HOV lane and are driven onto or off from the automated lane automatically. Those not equipped for automated lane-changing can use only the HOV lane for automated driving. Note that these vehicles have no access to the automated highway-to-highway connector ramps.

Negotiation among vehicles, with the support of the roadside intelligence, through communication for traffic merging at the merge point of the automated highway-tohighway interchange is assumed necessary for safety. Since all vehicles in the automated lane are under automated control at all times, such negotiation is possible. Note that such negotiation may not be possible if transition is allowed on the 

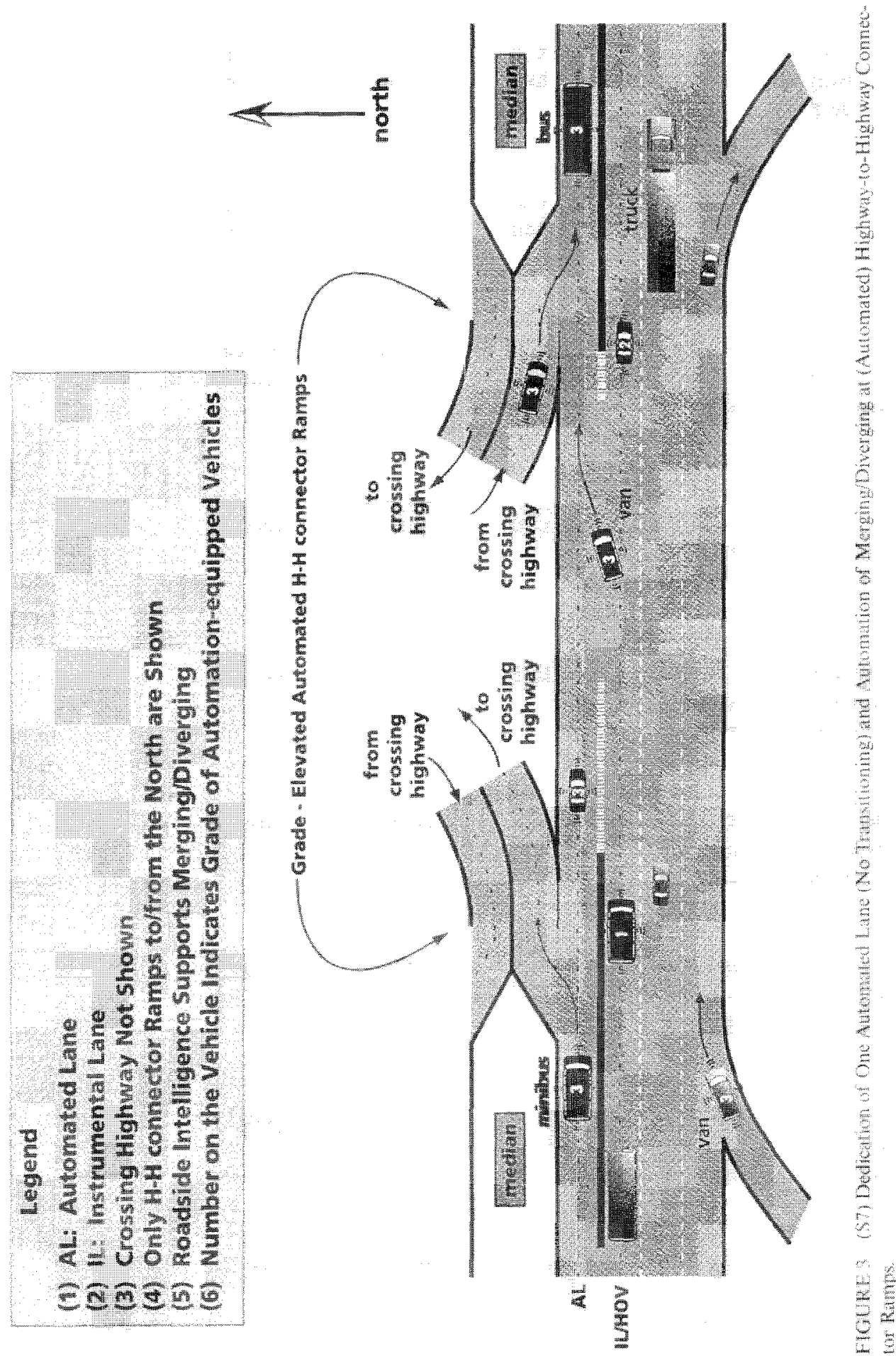
most lane, i.e. if the lane is still dedicated as an automated/transition lane. The reason is as follows. If a vehicle is approaching the left-most lane of a crossing highway from a highway-to-highway connector ramp but some vehicles near the merge point are still under manual control, negotiation is impossible. However, unlike a regular lane change, merging cannot wait, at least not as long as a regular change can, because it needs to take place at a specified location. This dilemma may be very dangerous. This motivated the strategy where automated merging is supported only when both traffic streams are under automatic control.

Note again the importance of the functional upgradability from the automated lane-changing capability to automated diverging/merging capability. (We have assumed that only the roadside needs upgrade.) At this stage, functional diversity encompasses non-fail-safe automated lane-cruising transit vehicle, fail-safe automated lane-cruising transit vehicles and automobiles, and fail-safe automated lane-changing/ merging/diverging transit vehicles and automobiles.

(S8) Construction of Automated On-Ramps and Off-Ramps with Barriers at HighDemand Locations. With sufficient and increasing demand, construction of automated on-ramps and off-ramps connecting city streets directly with the automated lanes adjacent to the median, especially at busy locations, begins. This supports fully automated driving from any automated on-ramp to any automated off-ramp. More importantly, it increases access and egress capacity at these high-demand locations. Vehicles equipped with automated lane-changing/merging/diverging capability access and egress the automated lane through the automated on-ramps/off-ramps, where available. Physical barriers separating the automated lane from the HOV lane at the highway's merge point with an automated on-ramp are erected for safety. Vehicles without automated lane-changing/merging/diverging capability can only access and egress the automated lane where barriers are absent. Automated lane-cruising vehicles can use the HOV/transition lane only. Although the cost of a highway-to-citystreet interchange may be moderate, there may be a large number of such busy locations implying the necessity of a large total capital investment and potential financing problems. The rate of construction could be slow.

(S9) Segregation of Automated Traffic from Manual Traffic with Physical Barriers for Safer and High-Speed Automated Driving. This stage is marked by the segregation of the automated lane from the manual traffic by erecting physical barriers between the automated lane and the HOV lane. See Figure 4 for illustration. Segregation is motivated by safety, higher-speed automated driving, high density and hence high capacity, and a driverless transit vehicle operation on the automated lane. Note that such barriers have already been erected at highway-to-highway interchanges as well as highway-to-street interchanges. Due to the segregation, the possibility of spill-over of traffic accidents from the manual traffic is minimized. Therefore, given the fail-safe feature of the automated lane-changing/merging/diverging vehicles, the previously virtually care-free driving is upgraded to completely care-free driving, i.e. hands-off, feet-off and "brain-off." (See description of Stage (S3).)

Such physical segregation essentially establishes a separate automated highway network system, possibly with convenient access from and egress to the "back-to- 


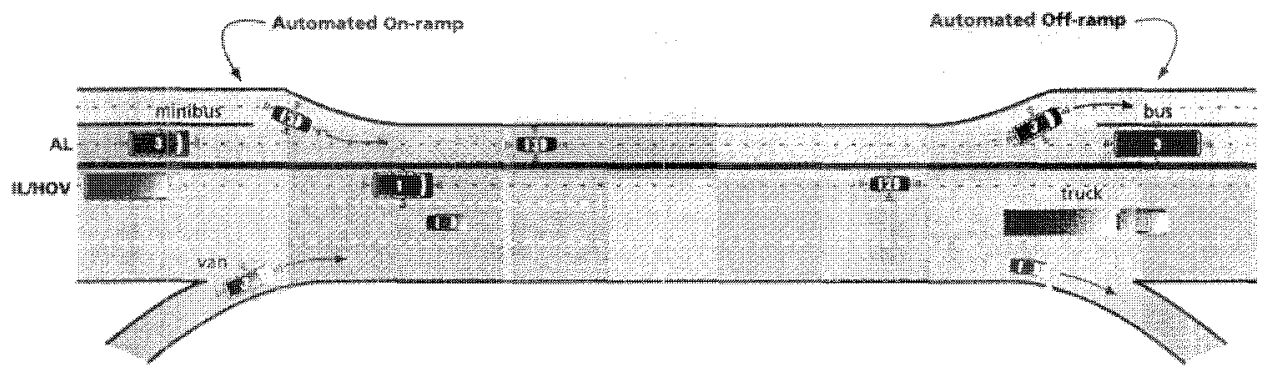

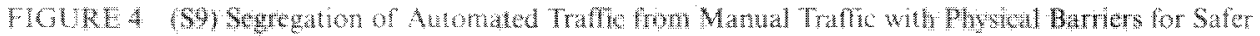
and High-sped Automated Driving

back" conventional network for manual tratic. After such a segregation, only those vehicles equipped with automated lane-changing/merging/diverging capability can use the segregated automated lanes. Those not so equipped can still use the HOV lane for antomated driving, but without access to the automated highway-10-highway connector ramps. Note again the importance of functional upgradability.

(510) Two Automated Lanes for Caparty and Higher Speted on the Second Auto mated Lane. With even higher demand, the HOV lane can be redesignated as an additional automated lane and the adjacent manual lane can be redesignated as the HOV lane. This is to increase the capacity and to accommodate higher speed on the second automated lane. Note that all vehicles ate loners so far.

(S1) Automobile Platooning on the Second Atwomated Lane for Higher Capacity During this stage addition of a second automated lane continues. More importantly, automobile platooning begins to be supported where and when higher capacity is needed. Note again the importance of fenctional upgradability. At this stage, lane usage is as follows. Automated lane-cruising loner vehicles, fail-safe or not, use only the HOV lane outside of the segregated automated lanes and have no access to any drect automated highway-to-highway connector ramps. All automated lane-changing/merging/diverging (fail-safe) vehicles, loner or platooning equipped, can use at all times the frst automated lane (i.e. one that interfaces with the automated onjor ramps). Automated platooning-equipped vehicles, when so desired by the driver, can use the sceond automated lane at all times. At congested locations and during congestion time. only the platooning equipred automobiles can use the second automated lane and they travel in platoons. On those sections where only one automated lane is avalable and when and where higher lane capacity is needed, "spontaneous platooning" is required of the platooning-equipped automobiles.

Possible issues related to platooning inelude user discomfort about the short spacing, liability and insurance (new laws possibly required for liability distribution), and environmental impact (due to the potentially large volume of traffic being supported through platooning). 
(S12) A Mature Urban AHS: A Physically Segregated Automated Highway Network Mainly on Existing Right-of-Way. By now, the evolution has reached the mature urban segregated AHS network described in detail at the beginning of this section.

\section{CONCLUSION}

On the highest level, the process of AHS deployment can be viewed as overcoming various difficulties in exchange for the provision of desirable AHS functions and user services. An evolutionary stage towards a mature AHS was defined as any discernible incremental functional step whose realization may encounter considerable difficulties requiring a significant amount of conscious effort to overcome. A good evolutionary scenario consists of stages each of which provides sufficient additional functionality and user services that justify the required effort to overcome the associated difficulties. Deployment of AHS technologies could be difficult. Six dimensions of deployment difficulties, namely technology, infrastructure, human factors, vehicle manufacturing and maintenance, insurance and public will, as well as specific difficulties were identified.

Note that issues regarding deploying AHS in the real world are not confined to the deployment stage but actually dictate AHS technological requirements. We now briefly illustrate that deployment could introduce many challenging R\&D issues, technological and otherwise. Consider the mature urban AHS defined in Section 4, where vehicle movements and maneuvers, and even the roadway, are under tight AHS monitoring and control without driver intervention. Through communication and sensing, uncertainty is minimized and safety can be achieved, at least in theory. However, if automated vehicles will need to be mixed with manually driven vehicles, either in the same lane or in an adjacent but non-physically-separated lane, in any of the intermediate deployment stages, then safety during any such stage may require ultra-sophisticated and yet reliable technologies than otherwise. Therefore, in this case, it is deployment, rather than the target mature AHS, that actually dictates the required technological sophistication. In the opinion of the author, deployment, if not properly treated at the outset of AHS R\&D, could become a potential "showstopper." Therefore, he invites intense research into deployment as an integral part of AHS system definition and specification.

Initial AHS market penetration could be the most difficult stage of all. Comparison of the desirability of the different mature AHS is also difficult, at least at this time. Given a feasible initial AHS deployment strategy and a target mature AHS, an evolutionary scenario can be viewed as a collection of intermediate stages, possibly overlapping and parallel, connecting the two ends. For illustration, the initial deployment strategy recently proposed by Tsao was adopted and a particular mature AHS was selected as an example. An evolutionary scenario consisting of a sequence of stages connecting the two ends was defined. The functional increments and the difficulties associated with each step were also discussed.

Note that there are many other possible mature AHS. This paper investigated only one alternative. The evolutionary scenario has been developed for illustration only and is not being advocated as the best, or even a viable, deployment strategy. In fact, 
the feasibility of any deployment strategy cannot be asserted with certainty at this conceptual level and much more study is required. Some of the deployment difficulties associated with this particular scenario may apply to other evolutionary scenarios. Such scenarios clarify what the deployment difficulties may be and hint when the difficulties might occur.

Development and evaluation of AHS evolutionary scenarios is a difficult task. Because of the large number of possible mature as well as evolutionary AHS scenarios, judgements based on preliminary analysis may have to be made to (i) gauge the desirability of the functions provided by the individual stages of the evolutionary scenarios, (ii) measure the associated difficulties and the required effort, and then (iii) select a manageable collection of good evolutionary scenarios. Detailed analyses, evaluations, and comparisons can then follow so that a small number of superior ones can be identified. The author invites more research on identifying AHS deployment difficulties as well as on designing and comparing AHS evolutionary scenarios.

\section{ACKNOWLEDGEMENT}

This work was performed as part of the US Department of Transportation Federal Highway Administration Contract Number DTFH61-93-C-00194 (AHS Precursor Systems Analyses-Delco Team). The author would like to thank two anonymous referees and Dr. Steven Shladover of the PATH Program for their valuable comments on an earlier version of this paper.

\section{REFERENCES}

Al-Ayat, R. and Hall, R.W., "A Conceptual Approach for Developing and Analyzing Alternate Evolutionary Deployment Strategies for Intelligent Vehicle/Highway Systems," PATH Program, Institute of Transportation Studies. University of California, Berkeley, UCB-ITS-PWP-94-05, 1994.

Bishop. J.R., McHale, G.M., and Stevens, W.B., "A Snap Shot of the Automated Highway Systems (AHS) Precursor Systems Analyses (PSA) Early Research Results." Proceedings of IVHS America Fourth Annual Meeting. Atlanta, Georgia, April, 1994.

Elias. J., Stuart. D.. Sweet. L and Kornhauser, A.. "Practicality of Automated Highway Systems, Volume I: Summary Report," Final Report FHWA-RD-79-39. Office of Research and Development, FHWA. US DOT, Washington, D.C.. 1977.

Federal Highway Administration. "Precursor Systems Analyses of Automated Highway Systems." (BAA) RFP No. DTFH6I-93-R-00047, 1992.

Hall. R.W. and Tsao. H.-S.J., "AHS Deployment: A Preliminary Assessment of Uncertainties." PATH Program. Institute of Transportation Studies. University of California, Berkeley, UCB-ITS-PWP94-02. 1994, to appear in Automated Highway Systems, ed. Ioannou. P.A., Society of Automotive Engineers.

Shladover. S. "Operation of Automated Guideway Transit Vehicles in Dynamically Reconfigured Trains and Platoons." (Extended Summary. Vol. I \& II). UMTA-MA-06-0085-79-1, UMTA-MA-06-008579-2 and UMTA-MA-06-0085-79-3. U.S. Department of Transportation. Urban Mass Transportation Administration, Washington. D.C.. July. 1979.

Stevens. W.B.. "The Automated Highway System (AHS) Concept Analysis." MITRE Research Report MTR-93W0000123. McLean, Virginia, 1993.

Stevens. W.B., "Goals and Definitions of Automated Highway System Concepts." Proceedings of IVHS America Fourth Annual Meeting. Atlanta. Georgia. April, 1994.

TRB (Transportation Research Board). Special Report 170 (Proceedings of a 1974 conference on Dual Mode Transportation), National Academy of Science. Washington. D.C.. 1976. 
Tsals, I., Boghani, A.B. and Greichen, J.J., "IVHS - Lessons from Other Industries," Proceedings of IVHS America Third Annual Meeting, Washington, D.C., April, 1993.

Tsao, H.-S.J., "Constraints on Initial AHS Deployment and the Concept Definition of a Shuttle Service for AHS Debut," IVHS Journal, Vol. 2(2), 159-173, 1995(a).

Tsao, H.-S.J., "A Staggered-Diamond Design for Automated/Manual-HOV Highway-to-Highway Interchanges," to appear in IVHS Journal, Vol. 2(3), 1995(b).

Tsao, H.-S.J, Hall, R.H. and Shladover, S.E., "Design Options for Operating Automated Highway Systems," Proceedings of Vehicle Navigation \& Information Systems Conference, Ottawa, Canada, Oct. 1993, 494-500, 1993(a).

Tsao, H.-S.J., Hall, R.W., and Shladover, S.E., Plocher, T.A. and Levitan, L.J., "Human Factors Design of Automated Highway Systems: First Generation Scenarios," FHWA Report No. FHWA-RD-93-123, Washington. D.C., 1993(b).

Tsao, H.-S.J., Plocher, T.A., Zhang, W.-B. and Shladover, S.E., "Human Factors Design of Automated Highway Systems: Second Generation Scenarios," in preparation, to be published as an FHWA Report, Washington. D.C., 1995.

Ward, J.D., "An Hypothesized Evolution of an Automated Highway System," draft report to the Rockwell International Corporation as part of Contract No. DTFH61-93-R00201 with the Federal Highway Administration, 1994. 